\title{
Incidences de la comparaison parallèle chez Du Bartas dans la réécriture de la Génèse
}

\section{Leila Lajevardi, Université Western Ontario}

L'étude que voici examinera l'emploi d'une figure de rhétorique dans La Sepmaine de Guillaume Salluste Du Bartas. Selon Bruno Braunrot, La Création du monde est un poème épique dans lequel l'auteur se livre à tous les jeux de langage qui constituent l'essentiel de la rhétorique baroque (378). Je propose une étude de la comparaison ou, plus précisément, ce qu'Alvin Emerson Creore appelle «parallel simile », que je traduirai par « la comparaison parallèle » (157). J'ai choisi de m'attarder sur cette figure car Du Bartas utilise les comparaisons d'une manière obsessionnelle dans La Sepmaine. Je détaillerai d'abord brièvement ce qu'est une comparaison, et expliciterai le fonctionnement d'une comparaison parallèle. J'examinerai ensuite quelques occurrences de cette figure dans le poème épique de Du Bartas, en suivant la chronologie du texte. J'avancerai pour finir une hypothèse sur le choix de la comparaison parallèle chez l'auteur.

La comparaison, figure de la ressemblance, effectue «un rapprochement fondé sur la perception d'une analogie entre deux réalités ou idées » par l'usage d'une copule comparative (Kokelberg 82). Cependant, il faut que ce rapprochement soit imprévu (Bacry 30). Un auteur utilise la comparaison parce qu'il « désire de mieux faire voir - ou de représenter avec plus de force - l'objet, l'idée, l'action ou la situation que l'on cherche à évoquer » (Kokelberg 82). Dans une comparaison, l'élément du texte qui fait l'objet de la comparaison est le comparé (ou thème) et le comparant (ou phore) est l'élément du texte qui effectue la comparaison (Klein-Lataud 72).

Dans son article intitulé «Ronsard, Du Bartas, and the Homeric Comparison », A. E. Creore décrit plusieurs types de comparaisons utilisés par Ronsard et Du Bartas. Contrairement aux pratiques rhétoriques de la poésie antique, Du Bartas fait usage fréquent de la comparaison parallèle (153). Cette figure exige que «each detail of the simile has a specific counterpart in the narration » (153). Creore constate que «while Du 
Bartas certainly learned the use of comparisons from Ronsard and the Pléiade, in the execution he gave them a flavor all his own » (156) :

The digressive similes in Du Bartas are far outnumbered by those of the parallel type which he customarily develops with great care. And not only is the parallelism considerably developed, but the length of the simile is frequently exactly the same as the passage of the narrative which it illustrates. (157)

L'appropriation de la comparaison parallèle par Du Bartas se caractérise ainsi par un certain raffinement.

Étudions maintenant quelques comparaisons parallèles bartasiennes. Dans ce long extrait du Premier Jour, l'auteur effectue l'une à la suite de l'autre deux comparaisons parallèles. Il compare d'abord Dieu à un auteur besogneux, penché sur sa table de travail. Il sollicite ensuite l'image d'un oiseau ou d'une poule qui couve ses œufs pour évoquer la création du monde par Dieu :

Ainsi qu'un bon esprit, qui grave sur l'autel

De la docte mémoire un ouvrage immortel,

En troupe, en table, au lict, tout jour, pour tout-jour vivre,

Discourt sur son discours, et nage sur son livre :

Ainsi l'Esprit de Dieu sembloit, en s'esbatant, Nager par le dessus de cet amas flottant.

(Autre soing ne veilloit pour lors dans sa poitrine :

Si le soing peut tomber en l'essence divine)

Ou bien comme l'oiseau qui tasche rendre vifs

Et ses œufs naturels, et ses œufs adoptifs,

Se tient couché sur eux, et d'une chaleur vive,

Fait qu'un rond jaune-blanc en un poulet s'avive :

D'une mesme façon l'Esprit de l'Eternel

Sembloit couver ce gouffre, et d'un soin paternel

Verser en chasque part une vertu feconde,

Pour d'un si lourd amas extraire un si beau monde. (I, 289-304)

La première comparaison parallèle s'étend sur les vers 289-296. Quatre vers décrivent d'abord l'auteur, ensuite un double point sert à marquer la fin de cette description. L'image de Dieu occupe ensuite les quatre prochains vers. Les copules comparatives sont «ainsi » au vers 289 et «ainsi... sembloit» au vers 293. Les deux instances de la copule comparative étant situées au début du vers, le parallélisme de la comparaison affecte jusqu'à la structure du poème. A chaque détail évoqué à propos l'auteur correspond un élément de la description de Dieu : 


\begin{tabular}{|l|l|}
\hline \multicolumn{1}{|c|}{ Description de l'auteur } & \multicolumn{1}{c|}{ Description de Dieu } \\
\hline un bon esprit & l'Esprit de Dieu \\
\hline qui grave sur l'autel & en s'esbatant \\
\hline nage sur son livre & Nager par le dessus de cet amas flottant. \\
\hline Discourt sur son discours & Autre soing ne veilloit \\
\hline
\end{tabular}

La deuxième comparaison parallèle s'étend sur les vers 297-304. Le tableau de l'oiseau qui couve ses œufs occupe quatre vers, tout comme la représentation de Dieu qui crée le monde. Les copules comparatives sont « ou bien comme » au vers 297 et « d'une mesme façon... sembloit » au vers 301 et 302. Elles sont encore une fois situées au début des vers. Le double point sert à articuler les deux comparaisons. Les correspondances sont organisées dans le tableau ci-dessous :

\begin{tabular}{|l|l|}
\hline \multicolumn{1}{|c|}{ Description de l'oiseau } & \multicolumn{1}{c|}{ Description de Dieu } \\
\hline l'oiseau & l'Esprit de l'Eternel \\
\hline qui tasche rendre vifs & Verser... une vertu feconde \\
\hline Et ses œufs naturels, et ses œufs adoptifs & en chasque part \\
\hline Se tient couché sur eux & couver ce gouffre, \\
\hline d'une chaleur vive & d'un soin paternel \\
\hline un rond jaune-blanc & un si lourd amas \\
\hline un poulet & un si beau monde. \\
\hline
\end{tabular}

Je postule que Du Bartas utilise des comparaisons parallèles pour rendre sensible le caractère abstrait du travail divin. En abordant l'abstraction à partir de sujets concrets et même quotidiens, le poète matérialise la figure de Dieu. En effet, le lecteur peut se faire une image de la narration dans son esprit grâce à la description et la profusion de détails que fournit l'auteur.

Dans cet extrait du Premier Jour, Du Bartas expose le façonnement de l'univers par Dieu en comparant le travail divin au travail de l'ourse :

De sagesse et pouvoir l'inespuisable source, En formant l'Univers, fit donq ainsi que l'ourse, Qui dans l'obscure grotte au bout de trente jours Une masse difforme enfante au lieu d'un ours : 
Et puis en la lechant, ores elle façonne

Ses deschirantes mains, or' sa teste felonne,

Or' ses pieds, or' son col : et d'un monceau si laid

Son industrie anime un animal parfait.

Car du vent de sa bouche ayant fait dans le Vuide

Un Tas confusement froid, ardant, sec, humide :

Par temps du Monde bas Dieu separe le haut :

Met à part peu à peu le chaud avec le chaud :

Renvoye le solide aveques le solide,

Le froid avec le froid, l'humide avec l'humide,

Autant qu'il est besoin : et forme ingenieux,

En six jours tous les corps de la terre et des cieux. (I, 407-422)

L'ourse façonnant son enfant occupe les huit premiers vers, les huit vers suivants étant consacrés à la création du monde. La copule comparative «ainsi que » apparaît au vers 408. Le point à la fin du vers 414 marque une césure entre les deux images. A chaque détail convoqué dans la première partie correspond un détail dans la suivante :

\begin{tabular}{|l|l|}
\hline \multicolumn{1}{|c|}{ Description de l'ourse } & \multicolumn{1}{c|}{ Description de Dieu } \\
\hline l'ourse & Dieu \\
\hline En la léchant & du vent de sa bouche \\
\hline dans l'obscure grotte & dans le Vuide \\
\hline au bout de trente jours & En six jours \\
\hline Une masse difforme & Un Tas confusement froid, ardant, sec, humide \\
\hline Son industrie anime & et forme ingenieux \\
\hline un animal parfait & tous les corps de la terre et des cieux. \\
\hline
\end{tabular}

Le lecteur n'a pas besoin d'avoir été témoin de la naissance d'un ours pour que cette image se rapporte dans son esprit à l'activité d'un Dieu créateur. Cet extrait nous démontre l'érudition et l'inventivité de Du Bartas, que L. Delaruelle souligne en retrouvant dans l'Histoire Naturelle de Pline l'Ancien une description similaire de l'ourse enfantant (339-340).

Un sujet scientifique qui a le potentiel d'être difficile à expliquer à ses lecteurs contemporains, est le fonctionnement des quatre éléments, mais Du Bartas entend expliquer «le grand livre du monde » ${ }^{1}$ à tous, y compris à «l'enfant qui n'a sept ans » et 
« le chassieux vieillard $»^{2}$. Je trouve cette comparaison parallèle au début du Second Jour, où sont amplement traités les quatre éléments :

Non que chaque Element en main porte tout-jour

D’un mesme corps le sceptre : ains regnant à son tour,

Il fait que le sujet dessous sa loy se renge,

Et que, changeant de roy, de naturel il change :

Comme sans respecter ni richesse ni sang

Chasque bon citoyen commande et sert de rang

Dans la libre cité, qui semble en peu d'espace,

Changeant de magistrat, changer aussi de face. (II, 75-82)

L'équilibre des quatre éléments dans le corps humain est comparé à la place des citoyens dans la cité. La copule comparative est «comme». Cette comparaison est par ailleurs reprise plusieurs fois dans le Second Jour pour expliquer le fonctionnement des quatre éléments. La description des éléments occupe quatre vers, suivi par quatre vers qui s'attachent au rôle des citoyens. Le double point marque le mi-chemin de la comparaison. Chaque détail évoqué dans la description des éléments correspond à un détail dans la description des citoyens :

\begin{tabular}{|l|l|}
\hline \multicolumn{1}{|c|}{ Description des éléments } & \multicolumn{1}{c|}{ Description des citoyens } \\
\hline chaque Element & Chasque bon citoyen \\
\hline d'un mesme corps & Dans la libre cité \\
\hline dessous sa loy se renge & commande et sert de rang \\
\hline changeant de roy & Changeant de magistrat \\
\hline de naturel il change & changer aussi de face. \\
\hline
\end{tabular}

Cette comparaison est une introduction au refrain (ou incantation) qui suit, où Du Bartas énumère les excès propres à chaque élément. Suivant mon hypothèse, Du Bartas utilise ici une comparaison parallèle pour aborder un sujet scientifique abstrait, comparant le fonctionnement des éléments à un sujet qui familier et accessible à son lecteur.

Dans cet extrait du Troisième Jour, Du Bartas expose au lecteur comment Dieu a fertilisé la terre par l'eau en comparant cette action avec celle du métallurgiste versant du plomb bouillant.

Voila donques comment la pesanteur de l'onde

Fit d'un oblique tour une isle de ce monde.

Car ainsi que le plomb que bouillant nous versons 
Dessus un corps esgal, coule en maintes façons, S'enfuit ici tout droit, là serpentant se joue, Ici son corps divise, et delà le renoue,

De ses chauds ruisselets presque en mesme moment

Dessus l'uni tableau toutes formes formant :

Dieu respandit les flots sur la terre feconde, En figure quarree, oblique, large, ronde,

En pyramide, en croix, pour au milieu de l'eau

Rendre nostre Univers et plus riche et plus beau. (III, 69-80)

On retrouve la copule comparative «ainsi que » au vers 71 . La description du plomb occupe six vers: des vers 71-76. Cependant, la description de l'eau sur la terre est tronquée en deux, occupant les vers 69-70 et ensuite 77-80. Ceci marque la première fois que Du Bartas divise sa comparaison de cette manière. Selon sa formule habituelle, dans une comparaison parallèle de douze vers comme celle-ci, les six premiers vers sont dédiés à un sujet et les six prochains au deuxième sujet. Le point au vers 70 introduit le comparant et le double point au vers 76 indique le retour du comparé. Chaque détail évoqué dans la description de l'eau reçoit un détail correspondant dans la description du plomb :

\begin{tabular}{|l|l|}
\hline \multicolumn{1}{|c|}{ Description de l'eau } & \multicolumn{1}{c|}{ Description du plomb } \\
\hline La pesanteur de l'onde & ainsi que le plomb bouillant \\
\hline Sur la terre feconde & Dessus un corps esgal \\
\hline Dieu répandit les flots & coule en maintes façons \\
\hline En figure quarree, oblique, large, ronde & Toutes formes formant \\
\hline Fit d'un oblique tour une île de ce monde & $\begin{array}{l}\text { Ici tout droit, là serpentant se joue,/Ici son } \\
\text { corps divise, et delà le renoue }\end{array}$ \\
\hline
\end{tabular}

Cette comparaison suggère au lecteur l'image de Dieu versant l'eau sur terre comme un métallurgiste versant du plomb sur une surface. L'avènement de l'eau sur terre résiste à la représentation mais en le comparant avec une activité concrète, Du Bartas confère une certaine transparence.

Dans son article, Creore commente ce passage tiré du Quatriesme Jour, où Du Bartas compare le Soleil à un prince. 
Eil du jour, si je di que tout ainsi qu'un Prince

Qui, plein de majesté, rode par sa province,

Est entouré de Ducs, de Comtes, de Barons,

Void derriere et devant marcher les escadrons

Des archers de sa garde, et n'a rien en sa bande

Qui sa sainte grandeur ne rende encor plus grande :

Toy de mesme rouant autour de l'Univers,

Qui ne vit que du feu de tes aspects divers,

Six grands Princes du ciel, trois devant, trois derriere,

Acompagnent, vassaux, ton char porte-lumiere :

Outre l'ost brillonnant du ciel plus haut monté :

Qui de toy ne reçoit pour solde que clarté. (IV, 519-530)

Les copules comparatives sont «tout ainsi que » et «toy de mesme ». Le comparé est le Soleil et le comparant est le prince : "The description of the prince occupies six verses, exactly the same number as that of the sun. The two parts are separated by a colon which acts as a sort of fulcrum to balance them. And within the two parts each detail of one is balanced by a detail of the other » (Creore 157). Les comparés et les comparants sont répartis dans le tableau ci-dessous ${ }^{3}$ :

\begin{tabular}{|l|l|}
\hline Description du Prince & Description du Soleil \\
\hline un prince & toy (le Soleil) \\
\hline plein de majesté & ne vit que du feu \\
\hline rode par sa province & rouant autour de l'univers \\
\hline entourré de ducs & six grands Princes... accompagnent \\
\hline derriere et devant & trois devant, trois derriere, \\
\hline escadrons des archers & l'ost brillonnant \\
\hline sainte grandeur & clarté \\
\hline
\end{tabular}

Du Bartas compare le Soleil à un prince pour mieux illustrer le fonctionnement du premier: en imaginant un prince accompagné par son entourage, les lecteurs de $L a$ Sepmaine peuvent imaginer comment le Soleil fait son parcours dans le $\operatorname{ciel}^{4}$.

Dans cet extrait du Quatrième Jour, Du Bartas comparer le Firmament à un paon afin d'imager l'encerclement de la terre par le Firmament.

Comme un paon, qui navré du piqueron d'amour,

Veut faire, piafard, à sa dame la cour,

Estaler tasche en rond les tresors de ses ailes 
Peinturees d'azur, marquetees d'estoilles, Rouant tout à l'entour d'un craquetant cerceau, Afin que son beau corps paroisse encor plus beau :

Le Firmament atteint d'une pareille flamme Desploye tous ses biens, rode autour de sa dame, Tend son rideau d'azur de jaune tavelé, Houpé de flocons d'or, d'ardans yeux piolé, Pommelé haut et bas de flambantes rouelles, Moucheté de clers feux, et parsemé d'estoilles, Pour faire que la terre aille plus ardemment Recevoir le doux fruict de son embrasement. (IV, 171-184)

Le paon rôdant autour de sa dame occupe les premiers six vers, ce qui laisse les derniers sept vers à la description du Firmament. Contrairement à la majorité des comparaisons parallèles, les descriptions sont ici de longueur inégale. La copule comparative est «comme » et le double point marque le mi-chemin de la comparaison. Chaque détail évoqué dans la description du paon correspond à un détail dans la description du Firmament :

\begin{tabular}{|l|l|}
\hline \multicolumn{1}{|c|}{ Le paon } & \multicolumn{1}{c|}{ Le ciel } \\
\hline comme un paon & Le Firmament \\
\hline navré du piqueron d'amour & atteint d'une pareille flamme \\
\hline Veut faire, piafard, à sa dame la cour & $\begin{array}{l}\text { Desploye tous ses biens, rode autour de sa } \\
\text { dame }\end{array}$ \\
\hline peinturees d'azur & Tend son rideau d'azur \\
\hline marquetees d'estoilles & parsemé d'estoilles \\
\hline $\begin{array}{l}\text { Estaler tasche en rond les tresors de ses } \\
\text { ailes }\end{array}$ & Pommelé haut et bas de flambantes rouelles \\
\hline
\end{tabular}

Du Bartas compare le Firmament à un paon pour mieux illustrer le parcours du premier : en imaginant un paon qui rode autour de sa dame, la manière dont le Firmament entoure la terre devient limpide au lecteur. Du Bartas reflète bien les actions du Firmament dans celles du paon : la couleur azur et l'image des étoiles figurent dans les deux cas.

Dans cet extrait du Sixième Jour, Du Bartas compare Dieu à un médecin (ou plus spécifiquement, à un chirurgien, quoique l'auteur n'utilise pas un terme aussi précis) : 
Comme le Medecin, qui desire trencher

Quelque membre incurable, avant que d'aprocher

Les glaives impiteux de la part offensee,

Endort le patient d'une boisson glacee,

Puis sans nulle douleur, guidé d'usage et d'art,

Pour sauver l'homme entier, il en coupe une part :

Le Tout-puissant ternit de nostre ayeul la face,

Verse dedans ses os une mortelle glace,

Sille ses yeux ardans d'un froid bandeau de fer, ${ }^{5}$

Guide presque ses pieds jusqu' au sueil de l'enfer,

Bref si bien engourdit et son corps et son ame,

Que sa chair sans douleur par ses flancs il entame,

Qu'il en tire une coste, et va d'elle formant

La mere des humains, gravant si dextrement

Tous les beaux traits d'Adam en la coste animee,

Qu'on ne peut discerner l'amant d'avec l'aimee. (VI, 961-976)

La copule comparative est «comme ». Le comparé est Dieu et le comparant est le médecin. La description du médecin occupe six vers, tandis que la description de Dieu occupe dix vers. Encore une fois, le double point sépare les deux descriptions. A chaque détail en correspond un autre :

\begin{tabular}{|l|l|}
\hline \multicolumn{1}{|c|}{ Dieu } & \multicolumn{1}{c|}{ Un médecin } \\
\hline Le Tout-Puissant & Comme le Medecin \\
\hline Verse dedans ses os une mortelle glace & Endort le patient d'une boisson glacée \\
\hline Que sa chair sans douleur & Puis sans nulle douleur \\
\hline Engourdit et son corps et son ame & Endort le patient \\
\hline $\begin{array}{l}\text { Que sa chair sans douleur par ses flancs il } \\
\text { entame }\end{array}$ & $\begin{array}{l}\text { Qui desire trencher / Quelque membre } \\
\text { incurable }\end{array}$ \\
\hline Il en tire une coste & il en coupe une part \\
\hline
\end{tabular}

Pour expliquer comment Dieu a pu prendre une côte d'Adam pour créer Ève, Du Bartas a eu l'ingénieuse idée de comparer cette action à un procédé familier à ses lecteurs, soit celui d'un médecin opérant un patient. Il réussit à matérialiser la création universelle dans la particularité d'un corps opéré. Cependant, Du Bartas semble momentanément s'être perdu dans son enthousiasme de la comparaison parce qu'il passe trois vers à expliquer comment la «mortelle glace» endort Adam, ce qui met en évidence la longueur inégal des deux comparaisons (voir vers 969-971). 
La fin du Septième Jour de la Sepmaine marque le début d'un changement important dans le sujet et le ton des comparaisons parallèles bartasiennes. Les comparaisons sont souvent plus brèves, parfois d'une longueur totale de seulement six vers, qui comporte donc nettement moins de détails. Au lieu d'expliquer comment Dieu a créé un phénomène donné, ce sont les êtres humains qui sont comparés aux animaux dans leurs mœurs.

Dans cet extrait du Septième Jour, Du Bartas montre pourquoi il faut se garder de parler tout le temps. Il compare celui qui parle trop à des oies qui savent voyager sans faire de bruit :

Toy, que la liberté d'une langue indiscrète Precipite en danger, d'un frein prudent arreste Ton desbordé babil, ainsi que sages font Les oyes qui passant de Cilice le mont Portent et nuict et jour dans leur bouche criarde Pour un muet baillon une pierre qui garde Que des aigles du Nord les troupeaux ravissans Ne descouvrent le vol de tant d'oiseaux passans. (VII, 587-594)

La description de celui qui parle trop, occupe trois vers tandis que la description des oies occupe cinq vers. On peut ainsi reconstruire les associations que le poème met en scène :

\begin{tabular}{|l|l|}
\hline \multicolumn{1}{|c|}{ Celui qui parle trop } & \multicolumn{1}{c|}{ L'oie qui émet trop de cris } \\
\hline Toy & Les oyes \\
\hline $\begin{array}{l}\text { la liberté d'une langue indiscrète } \text { ou bien } \\
\text { Ton desbordé babil }\end{array}$ & leur bouche criarde \\
\hline Precipite en danger, & $\begin{array}{l}\text { Que les aigles... / Ne descouvrent le vol de } \\
\text { tant d'oiseaux passans. }\end{array}$ \\
\hline d'un frein prudent arreste & $\begin{array}{l}\text { Portent et nuict et jour... / Pour un muet } \\
\text { baillon une pierre }\end{array}$ \\
\hline
\end{tabular}

La comparaison parallèle vient ici appuyer l'importance de bien choisir ses mots. Au lieu de s'attarder sur un sujet scientifique abstrait, comme c'est le plus souvent le cas, Du Bartas soulève un contenu moral. Cependant, la constante d'un motif pédagogique transparaît. Du Bartas enseigne la valorisation d'un certain comportement. Il montre combien les oies sont sages de garder une pierre dans leur bouche pour ne pas crier et 
attirer l'attention des prédateurs. Les êtres humains devraient se garder de parler tout le temps parce que, par inattention, ils peuvent dire quelque chose qui déplaît à autrui. Du Bartas louange la Création de Dieu en mettant en évidence cette caractéristique du comportement des oies.

Dans cet extrait du Septième Jour, Du Bartas compare le travail des religieux au travail du cœur humain :

Tout ainsi que le cœur un seul moment ne peut

Demeurer en repos, ains nuict et jour se meut,

Pour d'un ba-batement d'arteres en arteres

Envoyer haut et bas les esprits à ses frères :

Ceux à qui l'Eternel a commis son bercail

Doyvent estre tousjours en soin, veille et travail,

Pour souffler par leurs mœurs, et par doctrine exquise,

L'esprit vivifiant dans le corps de l'Eglise. (VII, 675-682)

La description du cœur occupe quatre vers suivi par quatre autres vers qui expriment le religieux dans le corps de l'église. Le double point à la fin du vers 678 indique la fin d'une description et le début de l'autre. Nous pouvons ranger les éléments de comparaison saillants dans le tableau ci-dessous :

\begin{tabular}{|l|l|}
\hline \multicolumn{1}{|c|}{ Le cœur } & \multicolumn{1}{c|}{ Les religieux } \\
\hline Le cœur & Ceux à qui l'Eternel a commis son bercail \\
\hline $\begin{array}{l}\text { un seul moment ne peut \ Demeurer en } \\
\text { repos, ains nuict et jour se meut }\end{array}$ & $\begin{array}{l}\text { Doyvent estre tousjours en soin, veille et } \\
\text { travail, }\end{array}$ \\
\hline Pour d'un ba-batement d'arteres en arteres & $\begin{array}{l}\text { Pour souffler par leurs mœurs, et par } \\
\text { doctrine exquise, }\end{array}$ \\
\hline Envoyer haut et bas les esprits à ses frères : & \begin{tabular}{l} 
L'esprit vivifiant dans le corps de l'Eglise. \\
\hline
\end{tabular}
\end{tabular}

Du Bartas utilise le fonctionnement du cœur pour indiquer que le travail des religieux ne doit cesser.

Pour conclure, j'estime que la comparaison parallèle est si fréquente qu'elle en vient à organiser la narration dans La Sepmaine. L'utilisation quasi obsessionnelle de cette figure de style semble révéler deux objectifs. Quoique mon étude ne soit pas exhaustive, la plupart des comparaisons parallèles que j'ai examinées dans les livres IVII, vise soit à éclairer le lecteur sur un sujet scientifique difficile à comprendre, soit à 
mieux illustrer le travail divin. En ce qui concerne le travail divin, Grahame Castor constate que, « in the sixteenth century divine creation was something mysterious and quite beyond the realm of man's comprehension » (91). Du Bartas utilise la comparaison parallèle dans le but de démystifier la création divine : elle contribue à clarifier des idées complexes pour le lecteur. En utilisant cette figure de style, Du Bartas rend les choses plus accessibles, crédibles et compréhensibles pour ses lecteurs. Creore constate que la comparaison parallèle bartasienne est étroitement liée à la narration, et que l'auteur utilise ce genre de comparaison dans un but didactique : «In Du Bartas it is abundantly clear that similes are used to make abstract ideas or abstruse scientific information more clear and more understandable to the reader»(158-159). En créant des comparaisons parallèles qui font appel aux sens physiques du lecteur, Du Bartas lui fait comprendre des concepts abstraits : «Furthermore, by extending the frame of reference, by multiplying the images through the medium of the extended comparison, Du Bartas even more than Ronsard is able to produce extraordinarily vivid visual, auditory, and even tactile images » (158-9). Grâce au caractère saisissant de ses comparaisons parallèles, l'auteur parvient à illustrer sa narration de manière vivifiante.

Quelques comparaisons parallèles du Septième Jour visent, non pas à éclairer le lecteur sur un sujet scientifique et abstrait, mais plutôt à transmettre un contenu moral. Du Bartas compare le comportement humain à un comportement animal ou organique (par exemple, le cœur) pour souligner les traits agréables et supérieurs et pour tenter d'élever les mœurs humaines. Bref, Du Bartas ouvre «le grand livre du monde ${ }^{6}$, il se tourne vers la nature, afin d'en tirer des leçons.

Les comparaisons parallèles de La Sepmaine ou Création du monde nous livrent une pensée rhétorique complexe. Dans une étude plus approfondie, j'aurais pu retenir les comparaisons parallèles dans un Jour unique de La Sepmaine ; il aurait aussi été possible d'étudier toutes les comparaisons parallèles qui portent sur un sujet en particulier, par exemple le soleil ou les éléments.

La comparaison parallèle sert de tremplin à Du Bartas pour montrer son érudition, son imagination et son enthousiasme pour le sujet de la Création divine (Creore 159). Il effectue une reprise de comparaisons traditionnelles qu'il rénove avec des inventions et des innovations (Dauphiné 127). Par l'usage qu'il fait de la rhétorique, l'auteur de la 
Création du monde décrit un univers inconnu au lecteur moderne: Du Bartas ouvre une fenêtre sur le monde, la culture et les modes de pensée de la Renaissance (Dagens 11 et Bellenger LII).

\section{Bibliographie}

Bacry, Patrick. Les figures de style et autres procédés stylistiques (coll. Sujets). Paris : Belin, 1992.

Braunrot, Bruno. «Une rhétorique de la surprise : motifs et figures de style dans la Création du Monde de Du Bartas ». Kentucky Romance Quarterly 4 (1973) : 371385 .

Castor, Grahame. Pléiade Poetics : A Study in Sixteenth-Century Thought and Terminology. Cambridge : Cambridge UP, 1964.

Creore, A. E. «Ronsard, Du Bartas, and the Homeric Comparison». Comparative Literature 3.2 (1951). 152-159.

Dagens, Jean. «Du Bartas, humaniste et encyclopédiste dévot ». Cahiers de l'Association internationale des études françaises 10 (1958). 9-24.

Dauphiné, James. «L'encyclopédisme poétique de Du Bartas». Du Bartas poète encyclopédique du XVIe siècle. Lyon, 1988. 121-130.

Delaruelle, L. «Recherches sur les sources de Du Bartas dans la Première Sepmaine ». Revue d'Histoire Littéraire de la France XL (1933). 321-354.

Du Bartas, Guillaume Salluste. La Sepmaine. Ed. établie, annotée et présentée par Yvonne Bellenger. Paris : Nizet, 1981.

Klein-Lataud, Christine. Précis des figures de style. Préface Alain Baudot (coll. Traduire). Écrire, Lire 2. Toronto : GREF, 1991.

Kokelberg, Jean. Les techniques du style: vocabulaire, figures de rhétorique, syntaxe, rythme. Paris (coll. Nathan-Université) : Nathan, 1991.

\footnotetext{
Notes

${ }^{1}$ La Sepmaine, Premier Jour, vers 151.

${ }^{2}$ La Sepmaine, Premier Jour, vers 169.

${ }^{3}$ Creore, 157.

${ }^{4}$ Il faut ajouter que cet extrait est précédé par plusieurs autres figures de style. Cette comparaison est en fait encadrée dans un hymne. Le vers 507 s'ouvre avec une apostrophe au Soleil, mais Du Bartas utilise des
} 
périphrases pour le désigner, tels que «Fontaine de chaleur » et «clair flambeau de ce Tout» (IV, 508, 509). Bruno Braunrot indique que La Sepmaine de Du Bartas est semé de périphrases dans le but de donner à deviner au lecteur (375-6). Qui plus est, la perfection du bien-dire à l'époque bartasienne consiste en l'usage fréquent des figures qui créent un «masque de mots» (Braunrot 374-5). Ensuite, aux vers 510-511, Du Bartas utilise le procédé du dubitatio. Cette figure est fréquemment utilisée dans les éloges royaux pour souligner l'abondance des richesses. Il y a une telle profusion d'éloges au Soleil que le poète ne sait par où commencer : « hé! di moy, par quel bout/Je doy prendre ton los?... » (IV, 510-511).

${ }^{5}$ La description de Dieu est plus longue que celle du médecin. Du Bartas, inspiré et enthousiasmé par l'image qu'il a créée, a embellit quelques détails, par exemple les effets de «la boisson glacee » ainsi que les beaux traits d'Ève.

${ }^{6}$ La Sepmaine, Premier Jour, vers 151. 